\title{
Evre Uyumlu Optik OFDM Sistemler için Karmaşık Aşırı Öğrenme Makinası Tabanlı Doğrusal Olmayan Denkleştirici
}

\author{
Ahmet Güner ${ }^{*}$ \\ ${ }^{1}$ Bingöl Üniversitesi, Mühendislik ve Mimarlık Fakültesi, Elektrik-Elektronik Mühendisliği Bölümü, Bingöl, Türkiye, (ORCID: 0000-0003-4073-7931), \\ aguner@bingol.edu.tr
}

(İlk Geliş Tarihi 27 Ağustos 2021 ve Kabul Tarihi 24 Aralık 2021)

(DOI: $10.31590 /$ ejosat.987797)

ATIF/REFERENCE: Güner, A. (2022). Evre Uyumlu Optik OFDM Sistemler için Karmaşık Aşırı Öğrenme Makinası Tabanlı Doğrusal Olmayan Denkleştirici. Avrupa Bilim ve Teknoloji Dergisi, (33), 26-31

$\ddot{\mathbf{O z}}$

Radio-over-Fiber (RoF), 5G ve ötesi sistemlerde daha uzun iletişim mesafelerinde daha hızlı veri iletimi için alternatif bir çözüm olarak sunulmaktadır. RoF'un alt bileşenlerinden birisi olan optik haberleşme sistemlerinde evre uyumlu alıcıların kullanımı önemli kazanımlar sağlamaktadır. Bu çalışmada MQAM evre uyumlu-OFDM alıcılar için iletişim kanalının doğrusal ve doğrusal olmayan etkilerini kompanze etmek amacıyla karmaşık-Aşırı Öğrenme Makinesi (K-AÖM) tabanlı doğrusal olmayan denkleştirici önerilmiş ve Monte Carlo benzetimleri ile performans analizleri yapılmıştır.

Anahtar Kelimeler: Karmaşık Aşırı Öğrenme Makinası, evre uyumlu optik OFDM, fiber doğrusal olmayan etki.

\section{Complex Extreme Learning Machine-based Nonlinear Equalizer for Coherent Optical OFDM Systems}

\begin{abstract}
Radio-over-Fiber (RoF) is offered as an alternative solution for faster data transmission over longer transmission distances in 5G and beyond systems. The use of coherent receivers provides significant gains in optical communication systems which is one of the subcomponents of RoF. In this paper, a complex Extreme Learning Machine-based nonlinear equalizer was proposed to compensate for linear and nonlinear effects of the transmission channel for MQAM coherent-OFDM receivers, and performance analyzes were performed with Monte Carlo simulations.
\end{abstract}

Keywords: Complex Extreme Learning Machine, coherent optical OFDM, fiber nonlinearity.

\footnotetext{
* Sorumlu Yazar: aguner@bingol.edu.tr
} 


\section{Giriş}

Günümüz teknolojisinde, artan taleplerin sonucunda veri iletişim kapasitesinin ve hızının artırılabildiği yeni haberleşme teknolojilerine ihtiyaç duyulmaktadır. Radio-over-Fiber (RoF) sistemleri hem $5 \mathrm{G}$ hem de ötesi için alternatif bir çözüm olarak araştırmacılar tarafindan ele alınmaktadır. RoF sistemlerinde, kablosuz ve optik haberleşme sistemlerinin birleştirilmesiyle kapasite ve hareketliliğin genişletilebildiği güçlü çözümler sunulmaktadır. RoF sistemlerinin alt bileşeni olan optik haberleşme sistemleri ile daha uzun mesafelere daha hızlı veri iletişim imkanı sağlanmaktadır (Jia et al., 2015).

Optik haberleşme sistemlerinde evre uyumlu (coherent) alıcıların kullanılması ile optik haberleşmeye olan ilgi daha fazla artmıştır (Kikuchi 2016). Çünkü evre uyumlu haberleşmedeki asıl amaç, alıcının yeteneklerini geliştirmek ve böylelikle daha uzun mesafelerde haberleşme gerçekleştirebilmektir. Örneğin, algılama yeteneğini arttırmaya yönelik genlik ve fazın aynı anda algılanması ve ileri düzey modülasyon biçimlerinin kolaylık adapte edilebilmesi sadece bir kaçıdır (Torres-Zugoide et al., 2016).

OFDM, yüksek spektral verimlilik sağlayan ve semboller arası girişime karşı dayanıklı çok taşıyıcılı modülasyon biçimidir. Evre uyumlu optik OFDM sistemler, hem evre uyumlu optik alıcıların ve hemde OFDM tekniğinin avantajlarını kullanabilmektedir (Yazgan et al., 2014).

Evre uyumlu optik OFDM sistemlerinde, OFDM tekniğinin semboller arası girişimin oluştuğu durumlarda sahip olduğu avantaj sayesinde, optik kanallardaki kromatik dispersiyon (CD) ve polarizasyon-mod dispersiyon (PMD) gibi doğrusal bozucu etkiler denkleştirici kullanılarak kolayca düzeltilebilmektedir.

Evre uyumlu optik OFDM sistemler, optik kanalın doğrusal ve doğrusal olmayan bozucu etkilerini alıcıda kompanze etmek için denkleştirici kullanımına imkan sağlamaktadır. Özellikle doğrusal olmayan bozucu etkiyi kompanze etmek için farklı sayısal sinyal işleme teknikleri (özellikle doğrusal olmayan denkleştiriciler) üzerine çalışılmaktadır. Örneğin, sayısal geri yayılım algoritması yoğun FFT kullanmasından dolayı fazla hesaplama karmaşıklığ gerektirmektedir (Gao et al., 2013). Dispersiyon ve doğrusal olmayan bozucu etkileri düzeltmek için öncesinde ve sonrasında düzeltme (pre-and post-compensation) tekniği kullanılmıştır (Lowery 2007). Bu teknik hem vericide (öncesi) hemde alıcıda (sonrası) kullanılmasından dolayı işlem karmaşıklığı gerektirmektedir. Volterra modeli doğrusal olmayan sinyal işlemede en yaygın kullanılan tekniktir (Giacoumidis et al., 2014). Bu tekniğin en büyük dezavantajı diğerleri gibi işlem yüküdür. Yapay sinir ağları, doğrusal olmayan karar sınırlarına sahip olmasından dolayı doğrusal olmayan bozucu etkileri düzeltmek için kullanılan bir başka yöntemdir (Jarajreh et al., 2015). YSA yöntemi, diğerleriyle karşılaştırıldığında daha az hesaplama karmaşıklığına sahiptir.

Bu çalışmada, MQAM evre uyumlu optik OFDM sistemleri için Karmaşık Aşırı Öğrenme Makinası (K-AÖM) tabanlı doğrusal olmayan denkleştirici sunulmuştur. K-AÖM tek gizli katmanlı ileri beslemeli sinir ağına sahiptir ve çıkış katsayılarını analitik olarak hesaplamaktadır. Bu özelliklerinden dolayı hız ve daha iyi genelleme başarımı gibi üstünlükleri bulunmaktadır (Güner et al., 2016).

Çalışmanın 2. bölümünde, evre uyumlu optik OFDM sistemlerinin genel blok yapısı gösterilmekte ve sistemin işleyişi anlatılmaktadır. Daha sonra karmaşık Aşırı Öğrenme Makinesi tabanlı doğrusal olmayan denkleştirici ile frekans düzlemi doğrusal kanal denkleştirici yapıları hakkında bilgi verilmektedir. 3. bölümde benzetim çalışmalarında kullanılan teknikler ve parametrelerden bahsettiksen sonra elde edilen benzetim sonuçları sunulmaktadır.

\section{Materyal ve Metot}

\subsection{Evre Uyumlu Optik OFDM Sistemi}

Evre uyumlu optik OFDM sistemin blok yapısı içerisinde bulunan her bir alt bileşen Şekil 1'de gösterilmektedir. Evre uyumlu optik OFDM sisteminde ilk olarak 0 ve 1'lerden oluşan veriler, iletim sırasında kullanılacak semboller için seçilen MQAM modülasyon türüne göre eşleştirilerek veri setleri oluşturulur. Ters hızlı Fourier dönüşümü (IFFT) işleminden sonra OFDM sembolleri, iki IQ optik modülatör (Mach-Zehnder Modulator-MZM) ile elektriksel sinyalden optik sinyale dönüştürülür ve optik kanala iletilir.

Optik hat, benzer uzunluklara sahip birden çok fiberden oluşur. Mevcut optik ağlar ve fiber optik iletişim sistemleri, standart tek modlu fiber (standard single mode fiber-SSMF) temellidir. Optik kanalların zayıflatıcı etkisinden dolayı, optik kanal boyunca her bir bölünmüş uzunluk boyunca iletilen sinyalin hat zayıflamaları için erbiyum katkılı fiber kuvvetlendirici (erbium-doped fiber amplifier-EDFA) kullanılır.

Alınan optik sinyal, yerel osilatör lazer (LD2) ile optik sinyal $90^{\circ}$ optik hibrit kullanılarak elektriksel sinyale dönüştürülür. Sonra elektriksel sinyaller, FFT işlemiyle zaman düzleminden frekans düzlemine geçirilerek OFDM alt taşıyıcılarındaki veri setleri elde edilir. Daha sonra optik kanalın veri setleri üzerindeki bozucu etkilerinin giderilmesi için sinyal işleme yöntemleri kullanılır.

\subsection{Karmaşık Aşırı Öğrenme Makinası Tabanlı Doğrusal Olmayan Denkleştirici}

Karmaşık Aşırı Öğrenme Makinası (K-AÖM), tek gizli katmanlı ileri beslemeli sinir ağı için önerilen bir öğrenme algoritmasıdır. K-AÖM'nin giriş ağırlıkları ve gizli katman eşik değerleri geleneksel ileri beslemeli ağların aksine rastgele üretilir ve tüm süreç boyunca sabit kalır. K-AÖM'nin çıkış ağırlıkları ise analitik yolla hesaplanır. Bu yaklaşım ile hızlı öğrenme süreci gerçekleştirilir (Güner et al., 2016).

Verilen karmaşık değerli eğitim örnek serisinde $\mathrm{y}_{\mathrm{i}} \in \mathrm{C}^{\mathrm{n}}$ giriş verilerini ve $x_{i} \in C^{m}, i=1,2, \cdots, N$ çıkış verilerini ifade etmektedir. $M$ tane gizli katman nöron ve karmaşık aktivasyon fonksiyonlu $\mathrm{g}_{\mathrm{c}}(\mathrm{z})$ tek gizli katmanlı ileri beslemeli sinir ağının çıkışı;

$$
\sum_{k=1}^{M} \beta_{k} g_{c}\left(w_{k} \cdot y_{i}+b_{k}\right)=x_{i}, \quad i=1, \ldots, N
$$

Burada, $w_{k} \in C^{n}$ karmaşık giriş ağırlık sütun vektörünü, $\beta_{\mathrm{k}} \in \mathrm{C}^{\mathrm{m}}$ karmaşık çıkış katmanı ağırlık vektörünü, $\mathrm{b}_{\mathrm{k}} \in \mathrm{C}$ $k$ 'nıncı gizli sinir hücresinin karmaşık eşik değerini ve $x_{i}$ ise tek gizli katmanlı ileri beslemeli ağın çıkışını göstermektedir. $\left(w_{k} \cdot y_{i}\right), w_{k}$ ve $y_{i}$ 'nin içsel çarpımıdır. 


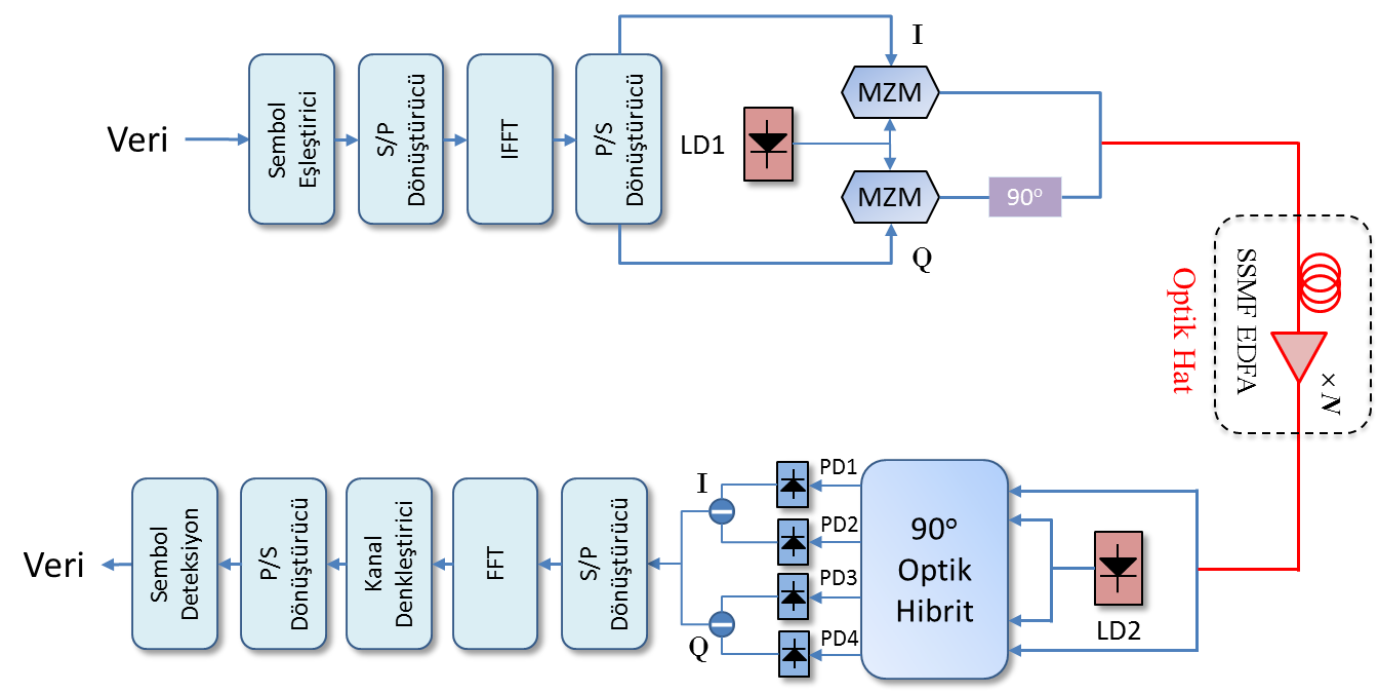

Şekil 1. Evre uyumlu Optik OFDM sisteminin blok gösterimi (Figure 1. Bock diagram of CoherentOpticak OFDM system).

Önerilen K-AÖM tabanlı doğrusal olmayan denkleştiricide, öncelikle eğitim dizileri kullanılarak $(\beta)$ karmaşık çıkış katmanı ağırlık vektörü hesaplanır. Eğitim dizisi verileri kullanılarak tek gizli katmanlı ileri beslemeli ağ çıkışı;

$$
\sum_{k=1}^{M} \beta_{k} g_{c}\left(w_{k} \cdot \tilde{y}_{i}+b_{k}\right)=\tilde{x}_{i}, \quad i=1, \ldots, N
$$

şeklinde yazılır. Burada $\tilde{y}$ alıcının girişindeki eğitim dizisi verisini, $\tilde{\mathrm{x}}$ orijinal eğitim dizisi verilerini ifade etmektedir. (2) denklemi $\mathrm{N}$ tane eşitlik matris formunda;

$$
\tilde{X}=H \beta
$$

olarak yazılabilir. Burada $H$ gizli katman çıkış matrisini, $\beta$ çıkış katmanı ağırlıklarını ve $\widetilde{\mathrm{X}}$ ise çıkış vektörünü ifade etmektedir ve $H, \beta$ ve $\widetilde{\mathrm{X}}$ ifadeleri (4) ve (5) denklemlerinde verilmiştir.

$$
\begin{gathered}
H=\left[\begin{array}{ccc}
g_{c}\left(w_{1} \cdot \tilde{y}_{1}+b_{1}\right) & \cdots & g_{c}\left(w_{M} \cdot \tilde{y}_{1}+b_{M}\right) \\
\vdots & \cdots & \vdots \\
g_{c}\left(w_{1} \cdot \tilde{y}_{N}+b_{1}\right) & \cdots & g_{c}\left(w_{M} \cdot \tilde{y}_{N}+b_{M}\right)
\end{array}\right]_{N \times M} \\
\beta=\left[\begin{array}{c}
\beta_{1}^{T} \\
\vdots \\
\beta_{M}^{T}
\end{array}\right]_{M \times 1} \quad \tilde{X}=\left[\begin{array}{c}
\tilde{x}_{1}^{T} \\
\vdots \\
\tilde{x}_{N}^{T}
\end{array}\right]_{N \times 1}
\end{gathered}
$$

K-AÖM de ağın eğitilmesi $\beta$ 'nın hesaplanması ile gerçekleştirilmektedir.

$$
\beta=H^{\dagger} \tilde{X}
$$

Burada $\mathrm{H}^{\dagger}, H^{\prime}$ 'n Moore Penrose genelleştirilmiş tersini göstermektedir ( $\mathrm{Li}$ et al., 2005). K-AÖM algoritması üç adım ile aşağıdaki gibi özetlenebilir;

adım 1. Rasgele olarak karmaşık giriş ağırlığı $\left(w_{k}\right)$ ve karmaşık eşik değeri $\left(b_{k}\right)$ seçilir.

adım 2. Karmaşık gizli katman çıkış matrisi $(H)$ hesaplanır.

adım 3. Karmaşık çıkış katmanı ağırlıkları $(\beta)$ (6) denklemi ile hesaplanir.
Daha sonra hesaplanan karmaşık çıkış katmanı ağırlıkları $(\beta)$ ve alınan veriler (y) kullanılarak (3) denklemi ile denkleştirme işlemi gerçekleştirilmektedir.

\subsection{Frekans Düzlemi Doğrusal Kanal Denkleştirici}

Çoklu-taşıyıcılı modülasyonlarda, her bir alt taşıyıcı için yüksek doğrulukla kanal kazancını kestirebilmek önemlidir. OFDM sistemlerinde, kanal katsayılarının kestiriminden sonra alınan sinyal üzerindeki bozucu etkileri kompanze etmek için genel olarak tek-taplı frekans düzlemi denkleştirici kullanılmaktadır.

$$
\hat{X}_{k}=\frac{Y_{k}}{\hat{H}_{k}}
$$

burada $k$ alt-taşıyıcı indeksi, $\hat{X}_{k}$ denkleştirilmiş sinyal, $\hat{H}_{k}$ k.alttaşıyıcıya karşılık gelen kestirilen kanal katsayısı ve $Y_{k}$ FFT işleminden sonra alınan sinyali ifade etmektedir.

$$
\hat{H}_{k}=\frac{Y_{T S k}}{X_{T S k}}
$$

FFT işleminden sonra kanal katsayıları (8) denklemi kullanılarak hesaplanmaktadır. Gerçekleştirilen işlem gönderilen ve alınan eğitim dizilerinin doğrusal olarak bir birlerine oranlanmasindan ibarettir. $\mathrm{Bu}$ nedenle sinyalin yayılımında oluşan doğrusal olmayan etkiler (7) denklemi ile gösterilen denkleştirici yapısı ile düzeltilememektedir.

\section{Araştırma Sonuçları ve Tartışma}

\subsection{Benzetim}

CO-OFDM haberleşme sistemi üzerinde optik kanal bozucu etkilerini araştırmak için Şekil 1'de gösterilen blokların MATLAB platformunda benzetimi oluşturuldu. Fiber içerisinde optik sinyal yayılımı, doğrusal olmayan Schrödinger denklemi (Non-Linear Schrödinger Equation-NLSE) ile modellenmektedir (Jiang et al., 2018).

$$
\frac{\partial A}{\partial z}+\frac{j \beta_{2}}{2} \frac{\partial^{2} A}{\partial t^{2}}+\frac{\alpha}{2} A=j \gamma A|A|^{2}
$$


burada $A$ sinyalin zarfi, $z$ iletim mesafesi, $\beta_{2}$ grup hiz yayılım parametresi, $\alpha$ zayıflatma katsayısı ve $\gamma$ doğrusal olmayan faktördür (Jiang et al., 2018). İletim kanal bloğunun benzetimi, bölünmüş adım Fourier yöntemi (Split-Step Fourier Method-SSFM) ile gerçekleştirildi. Optik haberleşme sistemlerinde bu yöntem, dispersiyon etkileri ve doğrusal olmayan bozucu etki gibi kanal bozulmalarının benzetimini gerçekleştirmek için birçok uygulama tarafından kullanılmaktadır (Ma et al., 2004). Tablo 1 ve Tablo 2'de, benzetimlerde kullanılan OFDM ve optik fiber parametrelerinin değerleri verilmektedir. K-AÖM için analitik yaklaşımla aktivasyon fonksiyonu $\operatorname{arcsinh}(\mathrm{z})=\int_{0}^{\mathrm{z}} \mathrm{dt} /\left[\left(1+\mathrm{t}^{2}\right)^{1 / 2}\right] \quad$ ve gizli katman nöron sayısı 20 olarak seçilmiştir.

Benzetim çalışmalarında, dispersiyon düzeltici fiber kullanılmamıştır. Fiber kablonun dispersiyon etkileri alıcıda sinyal işleme yöntemleriyle düzeltilmiştir.

\section{Tablo 1. OFDM Parametreleri (Table 1. OFDM Parameters)}

\begin{tabular}{|l|l|}
\hline Parametre & Değer \\
\hline FFT/IFFT uzunluğu $\left(N_{\mathrm{FFT}}\right)$ & 128 \\
\hline Alt taşıyıc sayısı & 80 \\
\hline Çevrimsel önek & $\% 6.25$ \\
\hline Modülasyon & QPSK,16QAM \\
\hline OFDM simge hızı & $39.06 \mathrm{MHz}$ \\
\hline
\end{tabular}

Tablo 2. Fiber Optik Parametreleri (Table 2. Fiber Optical Parameters)

\begin{tabular}{|l|l|}
\hline Parametre & Değer \\
\hline Dalga boyu $(\lambda)$ & $1550 \mathrm{~nm}$ \\
\hline $\begin{array}{l}\text { Kromatik dispersiyon } \\
\text { parametresi }\end{array}$ & $16 \mathrm{ps} /(\mathrm{nm} . \mathrm{km})$ \\
\hline PMD katsayısı & $0.1 \mathrm{ps} / \sqrt{\mathrm{km}}$ \\
\hline Doğrusal olmayan katsayısı $(\gamma)$ & $1.32(\mathrm{~W} \cdot \mathrm{km})^{-1}$ \\
\hline Zayıflatma $(\alpha)$ & $0.2 \mathrm{~dB} / \mathrm{km}$ \\
\hline EDFA gürültü oranı & $5 \mathrm{~dB}$ \\
\hline Bölünmüş uzunluk & $100 \mathrm{~km}$ \\
\hline Fiber & $\mathrm{SSMF}$ \\
\hline
\end{tabular}

Performans analizlerinde, bit hata oranı (bit error rate-BER) ve optik sinyal gürültü oranı (OSNR) performans ölçümü için kullanılmıştır. Optik SNR ile geçerli SNR arasındaki ilişki (10) denklemi ile ifade edilmektedir.

$$
\operatorname{OSNR}(d B)=10 \log _{10}[S N R]+10 \log _{10}\left(\frac{2 B_{f}}{R_{s}}\right)
$$

burada $B_{f}$ referans bant genişliğini (en yaygın olarak kullanılan referans bant genişliği $0.1 \mathrm{~nm}$ veya $12.5 \mathrm{GHz}$ 'dir) ve $R_{s}$ sembol oranıdir.

Benzetim çalışmaları, 100 bağımsız kanal üzerinden gerçekleştirildi. Gürültü, toplanır beyaz Gauss gürültüsü olarak modellendi. Verici tarafta, veri bitleri sözde rasgele ikili dizi (Pseudorandom Binary Sequence-PRBS) üreteci kullanılarak üretildi ve sonra QPSK ve 16QAM modülasyon türleri ile eşleştirildi. Benzetim çalışmalarında sistemin mükemmel olarak senkronize olduğu ve taşıyıcı frekans kayma etkisinin kompanze edildiği kabul edilmiştir. Benzetim çalışmalarında, fiber doğrusal olmayan bozucu etki, optik gürültü ve dispersiyon gibi optik haberleşme kanalına ait ve sinyalin yayılımında etkili olan parametreler dikkate alındı. Doğrusal bir etkiye sahip olan dispersiyon sinyalin sadece yoğunluğunu etkilemektedir. Fakat fiber doğrusal olmayan bozucu etki ise sadece sinyalin yoğunluğunu değil ayrıca fazını da etkilemektedir. Bu nedenle CO-OFDM haberleşme sistemlerinde amaçlanan doğrusal olmayan denkleştiricinin farklı senaryolar altında analizleri gerçekleştirildi.

\subsection{Benzetim Sonuçları ve Tartışma}

İlk olarak doğrusal olmayan etkiden daha çok dispersiyon etkisinin dikkate alındığ önceki doğrusal bölgedeki durum incelenmiştir.

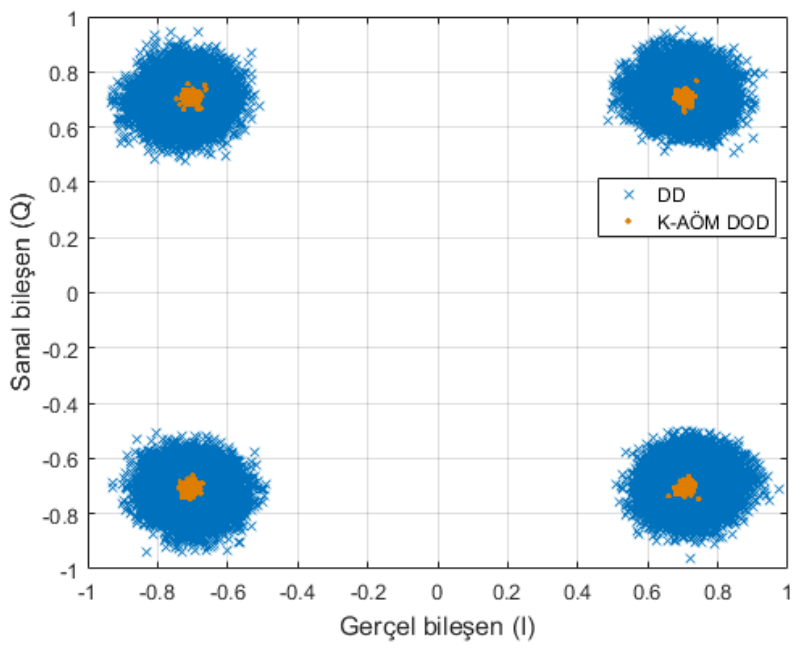

Şekil 2. $600 \mathrm{~km}$ iletimden sonra QPSK CO-OFDM sinyalinin denkleştirici çıkışındaki yıldız kümesi diyagramı (Figure 2. Output signal constellation of QPSK CO-OFDM after 600 $\mathrm{km}$ transmission)

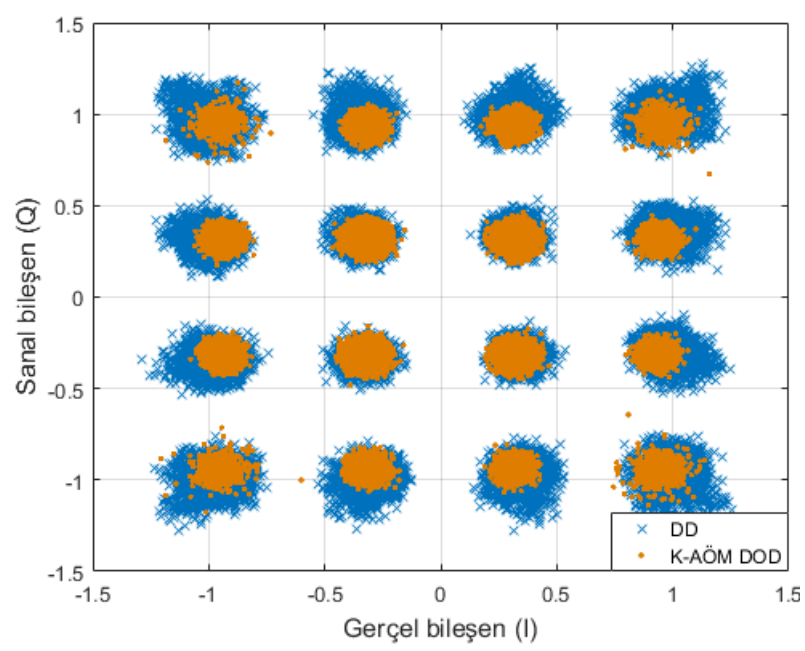

Şekil 3. $400 \mathrm{~km}$ iletimden sonra 16QAM CO-OFDM sinyalinin denkleştirici çıkışındaki yıldız kümesi diyagramı (Figure 3. Output signal constellation of 16QAM CO-OFDM after 400 km transmission)

Şekil 2 ve Şekil 3'de, QPSK ve 16QAM modülasyonlu OFDM sinyallerinin denkleştiricilerin çıkışındaki yıldız kümesi diyagramları gösterilmektedir. Yıldız kümesi diyagramı üzerindeki noktaların dağılımları incelendiğinde her iki denkleştiricinin dispersiyon etkisini düzelttiği iki şekilde de görülmektedir. Fakat doğrusal denkleştiriciyle (DD) karşılaştırıldığında, K-AÖM tabanlı doğrusal olmayan 
denkleştirici (K-AÖM DOD) dispersiyon etkisini azaltmakta ve yıldız kümesi diyagramı üzerindeki modülasyon türüne göre referans noktalara odaklanılmasında daha başarılı olduğu anlaşılmaktadır.

Fiber kablo uzunluğunun artırılmasıyla etkisi artan fiber doğrusal olmayan ve dispersiyon etkilerinin alınan sinyal üzerindeki ve denkleştirici çıkışındaki değişimleri Şekil 4 ve Şekil 5'de gösterilmektedir.

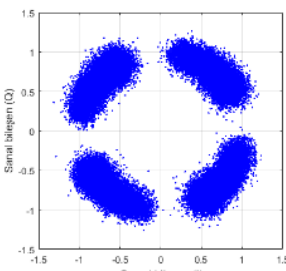

(a)

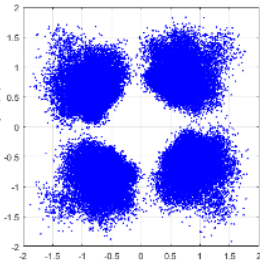

(b)

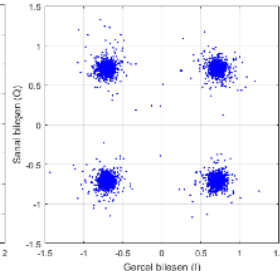

(c)
Şekil 4. $800 \mathrm{~km}$ iletimden sonra QPSK CO-OFDM sinyalinin (a) denkleştiriciden önce (b) DD sonra (c) K-AÖM DOD sonra (Figure 4. QPSK CO-OFDM signal after $800 \mathrm{~km}$ transmission (a) before equalizer (b) after DD (c) after $K$ $A O M D O D)$

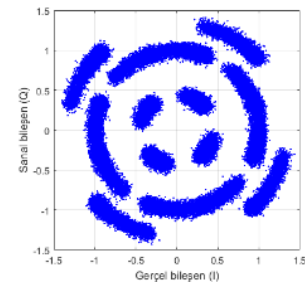

(a)

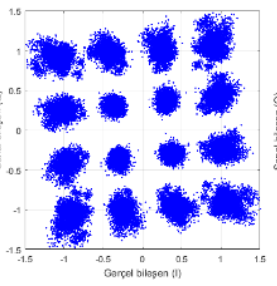

(b)

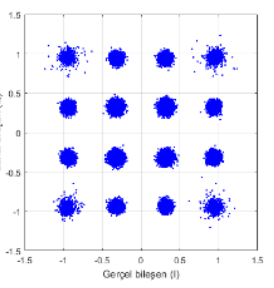

(c)
Şekil 5. $600 \mathrm{~km}$ iletimden sonra 16QAM CO-OFDM sinyalinin (a) denkleştiriciden önce (b) DD sonra (c) K-AÖM DOD sonra (Figure 5. 16QAM CO-OFDM signal after $600 \mathrm{~km}$ transmission (a) before equalizer $(b)$ after $D D(c)$ after $K$ $A O M D O D)$

Sırasıyla QPSK ve 16QAM için denkleştirici kullanılmadığı durumdaki alınan sinyale ait yıldız kümesi diyagramı üzerindeki noktalar Şekil 4.a ve Şekil 5.a'daki gibi bir dağılım göstermektedir. Fiber kablo uzunluğunun bir fonksiyonu olan dispersiyon ve fiber doğrusal olmayan bozucu etki, alınan sinyale ait yıldız kümesi noktalarının dairesel olarak yayılmasına ve saçılmasına neden olmaktadır. Doğrusal denkleştirici çıkışındaki sinyale ait yıldız kümesi diyagramı üzerindeki değişim Şekil 4.b ve Şekil 5.b'de gösterilmektedir. Alınan sinyal üzerindeki dispersiyon etkisi düzeltilebilmiş fakat fiber doğrusal olmayan bozucu etkiden dolayı oluşan saçılım etkisi tam olarak düzeltilememiştir. Önerilen denkleştirici çıkışındaki sinyale ait yıldız kümesi diyagramı üzerindeki değişim Şekil 4.c ve Şekil 5.c'de gösterilmektedir. Yıldız kümesi diyagramı üzerindeki noktaların değişimlerine dikkat ile bakıldığında, önerilen denkleştiricinin hem dispersiyon hem de doğrusal olmayan etkinin alınan sinyal üzerindeki etkilerini azaltma noktasında doğrusal denkleştiriciye göre daha başarılı olduğu gösterilmektedir.

Şekil 4 ve Şekil 5'deki değişimler denkleştiricilerin kullanımı ile daha uzun mesafelere güvenilir bir iletişimin gerçekleştirilebileceğinin göstergesidir. Bu sebepten dolayı Şekil 6 ve Şekil 7'de, önerilen denkleştirici ile doğrusal denkleştiricinin farklı fiber kablo uzunluklarındaki performans değişimleri gösterilmektedir. İletim kanalının bozucu etkilerine karşı önerilen denkleştiricinin doğrusal denkleştiriciye göre daha başarılı olmasından dolayı, önerilen denkleştirici ile daha uzun mesafelere güvenli iletişim imkanı mümkün olmaktadır. Örneğin, ileri hata düzeltme (forward error correction-FEC) sınırı olan BER $=10^{-3}$ değerine doğrusal denkleştirici ile QPSK ve 16QAM için yaklaşık 870 ve $560 \mathrm{~km}$ fiber kablo uzunluğunda ulaşılırken, önerilen denkleştirici ile yaklaşık 950 ve $850 \mathrm{~km}$ fiber kablo uzunluğunda ulaşılmaktadır.

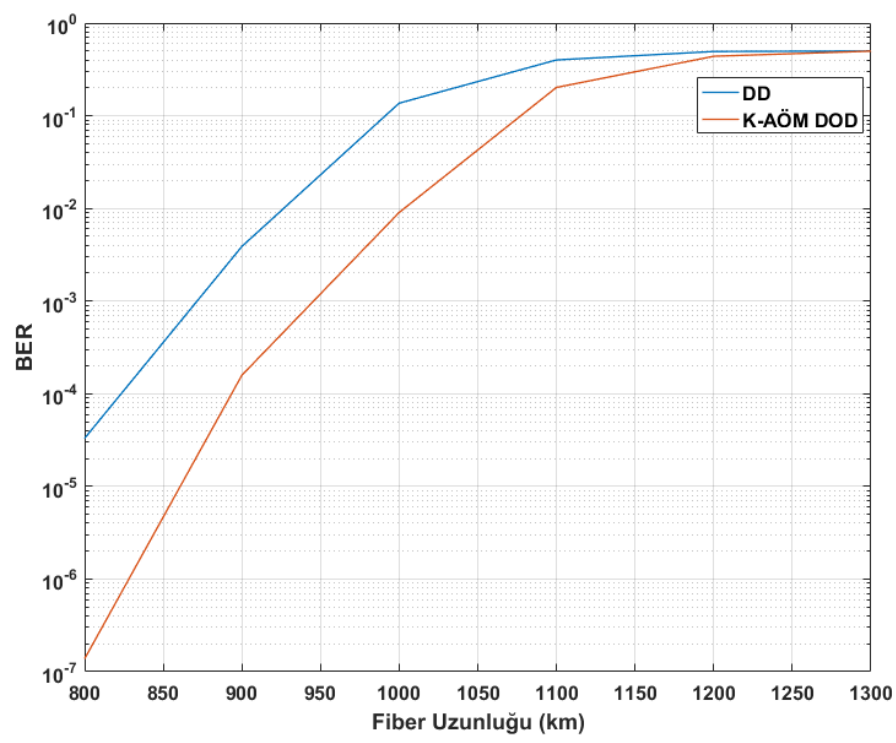

Şekil 6. QPSK için farklı fiber uzunluklara karşı kanal denkleştiricilerin BER başarımlarl (Figure 6. BER performances of channel equalizers different fiber lengths for QPSK)

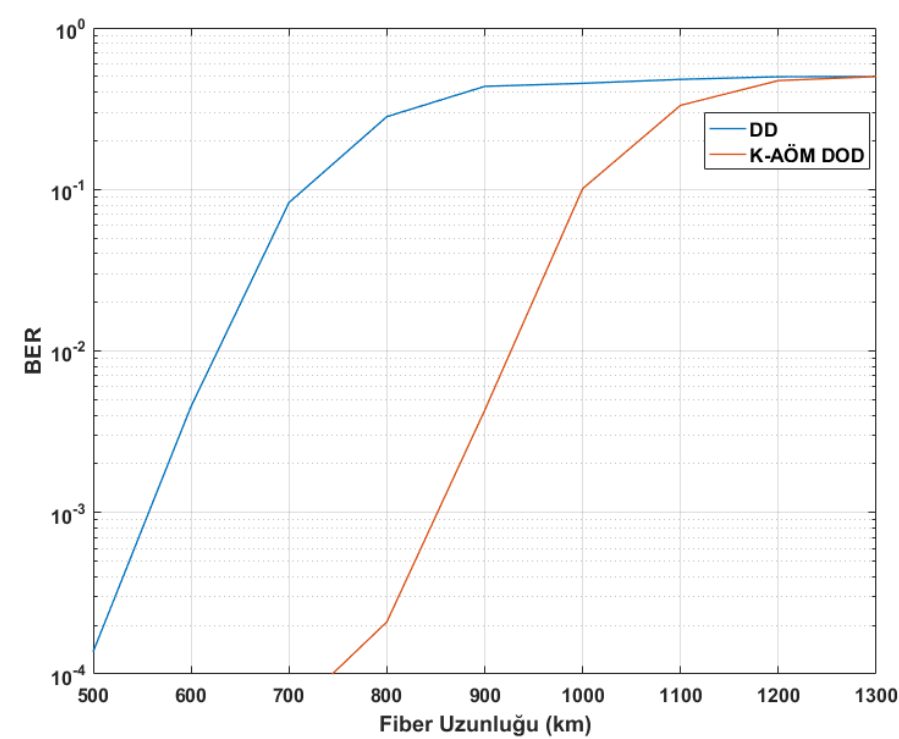

Şekil 7. 16QAM için farklı fiber uzunluklara karşı kanal denkleştiricilerin BER başarımları (Figure 7. BER performances of channel equalizers different fiber lengths for 16QAM)

Fiber doğrusal olmayan bozucu etkinin düşük olduğu bölgede, QPSK ve 16QAM için farklı optik gürültü seviyelerindeki BER performanslarının karşılaştırılması Şekil 8 ve Şekil 9'da gösterilmektedir. Denkleştiricileri 
karşılaştırdığımız zaman, FEC sınırı için önerilen denkleştiriciyle QPSK modülasyonunda yaklaşı $2 \mathrm{~dB}$ ve 16QAM modülasyonunda yaklaşık $3.5 \mathrm{~dB}$ 'lik bir kazanç elde edilmektedir.

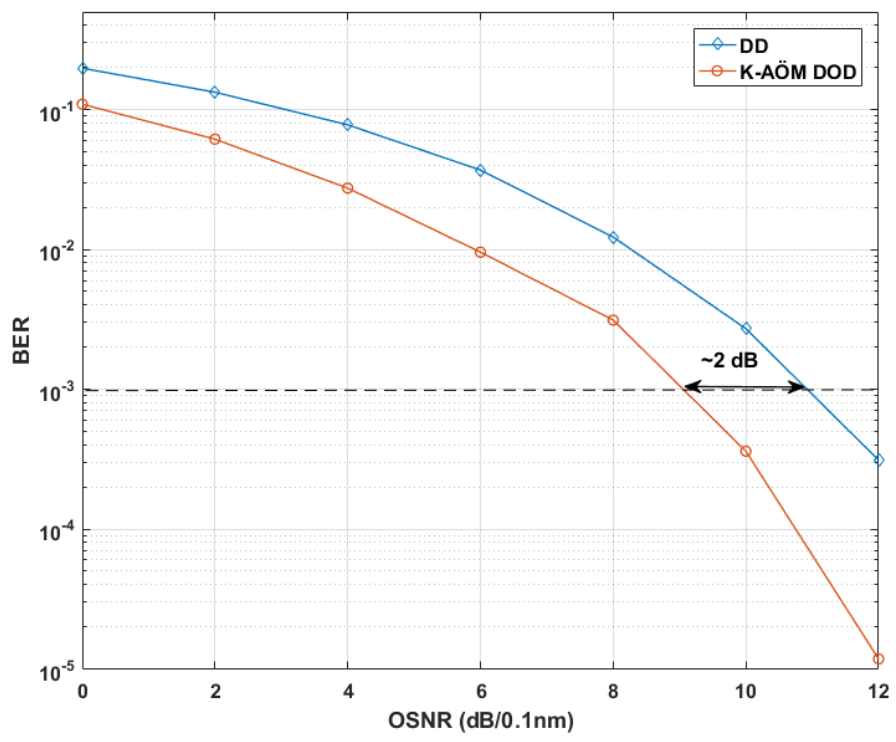

Şekil 8. $700 \mathrm{~km}$ iletimden sonra QPSK için OSNR değişimine karşı kanal denkleştiricilerin BER başarımları (Figure 8. BER performances of channel equalizers versus OSNR for QPSK after $700 \mathrm{~km}$ transmission)

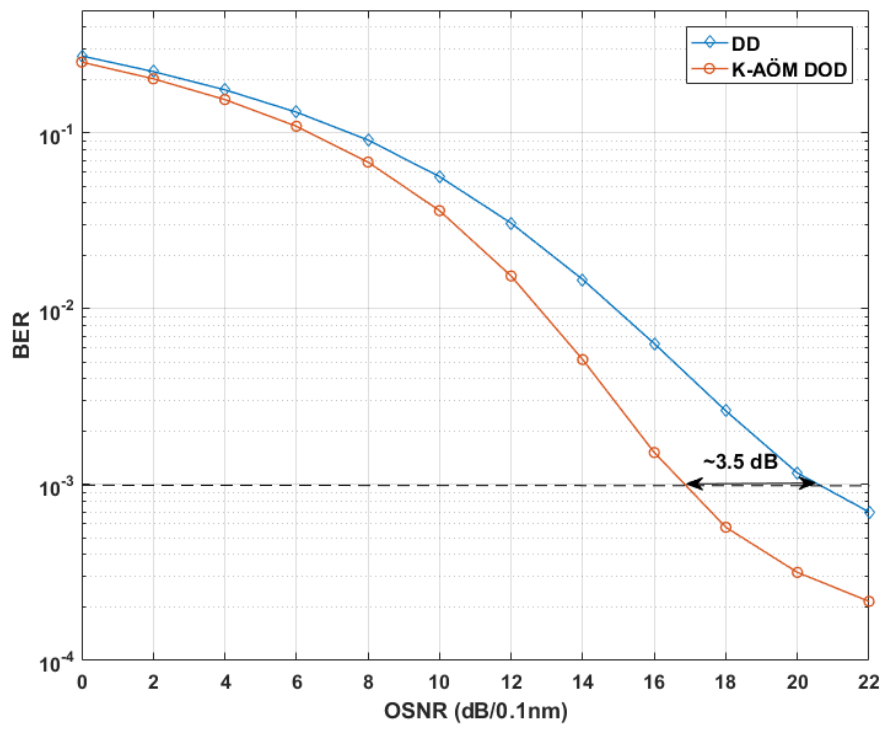

Şekil 9. $500 \mathrm{~km}$ iletimden sonra 16QAM için OSNR değişimine karşı kanal denkleştiricilerin BER başarımları (Figure 9. $B E R$ performances of channel equalizers versus OSNR for 16QAM after $500 \mathrm{~km}$ transmission)

\section{Sonuç}

Optik haberleşme sistemlerinde evre uyumlu OFDM alıcılar için makine öğrenme tekniği olan karmaşık Aşırı Öğrenme Makinası tabanlı doğrusal olmayan denkleştirici önerildi. KAÖM içerisinde karmaşık ve doğrusal olmayan aktivasyon fonksiyonunun kullanılması ile önerilen denkleştiricinin iletim kanalının doğrusal olmayan etkilerinin etkin olduğu durumlarda e-ISSN: 2148-2683 daha başarılı olduğu yapılan analizlerle gösterildi. OFDM sisteminde kullanılan klasik frekans düzlemi denkleştiricisi (doğrusal denkleştirici) ile önerilen denkleştirici, farklı iletişim mesafelerinde ve optik gürültü seviyelerinde karşılaştırıldığı zaman önerilen denkleştiricinin sağlamış olduğu avantajlar başarım eğrileriyle gösterildi.

\section{Kaynakça}

Jia Z., Yu J., and Chang G. K. (2006). A full-duplex radio-overfiber system based on optical carrier suppression andreuse. IEEE Photon. Technol. Lett., vol. 18, no. 16, pp. 1726-1728.

Kikuchi K. (2016). Fundamentals of coherent optical fiber communications. J. Lightw. Technol., vol. 34, no. 1, pp. 157179.

Torres-Zugaide J., Aldaya I., Campuzano G., and Castano G. (2016). Hammerstein-based equalizer for nonlinear compensation in coherent OFDM long-reach PONs. International Conference on Transparent Optical Networks (ICTON), pp. 1-3, 2016, Trento, Italy.

Yazgan A., and Çavdar İ.H. (2014). Optimum link distance determination for a constant signal to noise ratio in M-ary PSK modulated coherent optical OFDM systems. TELECOMMUNICATION SYSTEMS, vol. 55, pp. 461-470.

Gao G., Zhang J., and Gu W. (2013). Analytical evaluation of practical DBPbased intra-channel nonlinearity compensators. IEEE Photon. Technol. Lett., vol. 25, no. 8, pp. 717-720.

Lowery A. J. (2007). Fiber nonlinearity pre-and postcompensation for long-haul optical links using OFDM. Opt. Exp., vol. 15, no. 20, pp. 12965-12970.

Giacoumidis E., Aldaya I., Jarajreh M. A., Tsokanos A., Thai Le S., Farjady F., Jaouën Y., Ellis A. D., and Doran N. J. (2014). Volterra-Based Reconfigurable Nonlinear Equalizer for Coherent OFDM. IEEE Photonics Technology Letters, vol. 26, no. 14, pp. 1383-1386.

Jarajreh M.A., Giacoumidis E., Aldaya I., Le S.T., Tsokanos A., Ghassemlooy Z., and Doran N. J. (2015). Artificial neural network nonlinear equalizer for coherent optical OFDM. IEEE Photon. Technol. Lett., vol. 27, no. 4, pp. 387-390.

Güner A, Alçin Ö.F. and Üstündağ M. (2016). Feature Analysis of Time and Frequency Domain for Automatic Modulation Classification. International Artificial Intelligence and Data Processing Symposium, pp. 404-408, 2016, Malatya, Türkiye.

Li M.B., Huang G.B., Saratchandran P. and Sundararajan H. (2005). Fully complex extreme learning machine. Neurocomputing, vol. 68, pp. 306-314.

Jiang L., Yan L., Yi A., Pan Y., Hao M., Pan W., Luo B., and Jaouen Y. (2018). Chromatic Dispersion, Nonlinear Parameter, and Modulation Format Monitoring Based on Godard's Error for Coherent Optical Transmission Systems. IEEE Photonics Journal, vol. 10, no. 1, pp. 1-12.

Ma C. P. and Kuo J. W. (2004). Orthogonal frequency division multiplex with multi-level technology in optical storage application. Japanese Journal of Applied Physics, vol. 43, no. 4878 